\title{
Image Series
}

National Cancer Institute

\section{Source}

National Cancer Institute. Image Series. NCI Thesaurus. Code C69225.

A collection of images with a common element. 